\title{
Product Integrated Innovation Based on Function
}

\author{
Qinghai $\mathrm{Li}^{1}$, Guozhong $\mathrm{Cao}^{1}$, Haixia $\mathrm{Guo}^{2}$, and Jiang $\mathrm{Yu}^{3}$ \\ ${ }^{1}$ School of Mechanical Engineering, Hebei University of Technology, \\ 300130, Tianjin, China \\ ${ }^{2}$ Library of Hebei University of Technology, 300130, Tianjin, China \\ ${ }^{3}$ Hebei Energy Institute of Vocation and Technology, 063000, Tangshan, China \\ \{Qinghai.Li, Guozhong.Cao, Haixia.Guo, Yu Jiang, \\ caoguozhong\} @hebut. edu.cn
}

\begin{abstract}
Product innovation is a process of knowledge and technology resource integration. This paper mainly researches the process and realization of product integrated innovation based on function. Several function integrated methods are presented, and the principle solutions are selected and evaluated based on extend effect model. The process model for product integrated innovation is proposed, which provides a systematic approach to product integrated innovation from functional design angle. A design example for integrated innovation of Chinese medicine demonstrates the proposed method is feasible.
\end{abstract}

Keywords: Integrated innovation, function, effect.

\section{Introduction}

Product innovation is a process of knowledge and technology resource integration [1]. The concept of integrated innovation was put forward by Iansiti [2] firstly, and the integrated innovation was to adapt for the uncertainty of market and the rapid development of technology [3]. In recent years, integrated innovation has become one of the focuses of innovation management researching field [4-5], but the study of systematic method for product integrated innovation is still at the exploratory stage.

Product Innovation can be achieved by structure improvement, structure redesign, principle redesign or function redesign [5]. Product functional design is an important method for high-level innovation. First, the overall function is determined by user requirement. Then the overall function is broken down into a set of sub-functions and function units, and the function structure is composed of functions and flow of energy, materials, and signals. The principle solution is generated by the search for appropriate working principles and their combination [6].

The main focus of function design is to integrate function by user requirements and generate the principle solution that can fulfill the required functions. Researchers have different views on the way to establish function structure [6-7]. However, it is difficult to know when to stop decomposition and the process of functional decomposition mainly relies on designers' experience and expertise [8].

Function-to-structure mapping can be divided into two main categories: direct mapping and indirect mapping. Catalogue-driven approach [9-10] is common for the 
direct mapping, and Function-Behavior-Structure (FBS) [11-12] is common for indirect mapping. However, these methods do not talk about the law of mapping function-to-structure [13].

In this article, we focus on process and realization of product integrated innovation based on function. In Section 2 function integrated methods are presented. In the next section, the principle solutions are selected and evaluated based on extend effect model. In section 4 the product integrated innovation process model based on function is proposed. The design example is discussed in Section 5, and conclusions are drawn with implications for future work in Section 6.

\section{Function Integration}

Function is a statement to describe the transformation between input flow and output flow, aiming to achieve the designer's purpose. Function is the specification and abstract formulation of system from the viewpoint of technology. Functions can be represented by verb-noun pairs.

To satisfy the user requirements entirely, such as performance, cost, efficiency, maintenance, safety, deadline, etc., the overall function should be fulfilled by varies sub-functions, which are divided into main function(s), supporting functions and additional functions. Main function serves the overall function directly, and each system can be characterized by its main function. Supporting functions provide support to the execution of the system's main function(s). Additional functions are the functions that the system provides in addition to the main function(s).These functions can be integrated by following recommendations:

\section{Function Completeness}

Function completeness must ensure delivery of a general goal of the system and correspond with the functional purpose of the system. The realization of overall function should start from main function, and then select required auxiliary functions around main function. A viable complicated technical system consists of engine, transmission, executive element and control system. The main function of system is accomplished by executive element. Energy supply, transmission and control are the supporting functions of the system. To perform the overall function varies types of functions need to be integrated.

\section{Ideality of Function}

Technological systems evolve toward increasing system ideality. Ideality is defined as the ratio of a system's useful functions (UF) to its harmful functions (HF) [14].

$$
\text { Ideality }=\frac{\sum U F}{\sum H F}
$$

According to the formula above, there are at least two approaches to increasing system ideality: increase the number or magnitude of the useful functions; and reduce the cost, number or magnitude of the harmful functions. 
3. Modes of function integration

To increase the number or magnitude of the useful functions it is possible to find the existing functions and integrate them by the following modes:

- integrating the same functions

- integrating the similar functions

- integrating the opposite functions

- integrating the different functions

Consider integrating the systems that have same, similar, opposite or different functions, the functions of the new system may have the desired properties, may compensate for the shortcomings, or may be more precisely controlled.

Any technology of products is evolving in a biological pattern, meaning that it will go through four main stages. They are infancy, growth, maturity, and old (or decline) [15]. In infancy stage the new technical system is invented, and the overall function of system is achieved by function completeness. In growth stage the supporting functions and additional functions are added to the system by function integration to perform better functionality. The degree of ideality of technical system increases gradually. In maturity and old stages the performance index of the system approaches a limit, so the main goal is to reduce cost rather than improve function. These stages are plotted on the evolutionary "S-Curve" in Fig. 1.

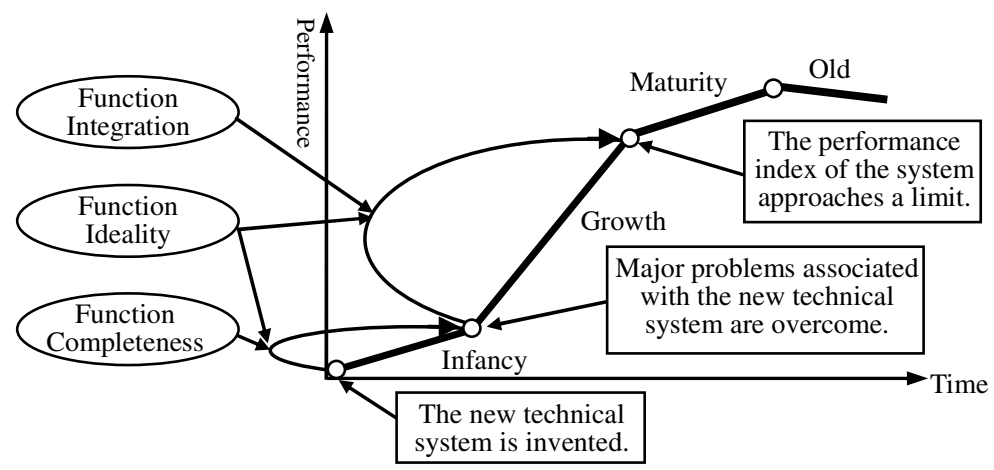

Fig. 1. Evolutionary S-curve of a system

The process of establishment of function structure is shown in Fig. 2. The first step is to determine the overall function according to the customer requirements. Then, several sub-functions are selected by function completeness. In order to increase system performance and improve existing problems in system the degree of ideality is measured and the auxiliary functions are integrated by reasonable combination and appropriate adjustment of sub-functions. In the end, the function structure is constructed. 


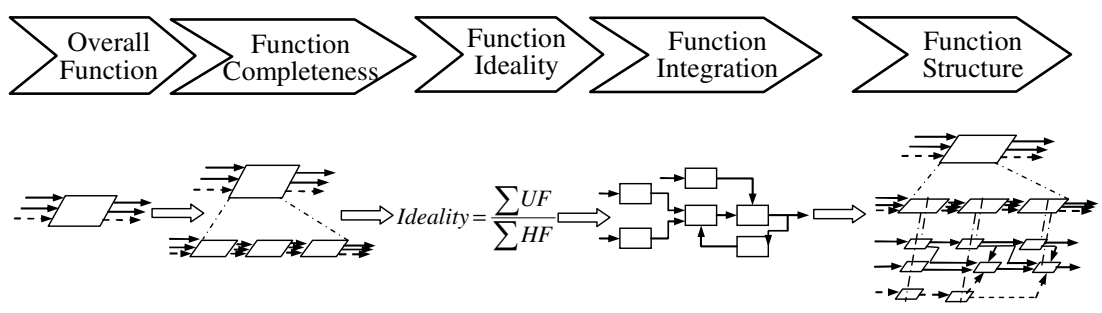

Fig. 2. The establishment of functional structure

\section{Function Realization}

Effect is one of the knowledge base tools in TRIZ. By the analysis of patents, effects are emerged from the relevance between functions delivered by a product described in a patent and a principle used in the product [16]. Studies of numerous patents indicate that strong inventive solutions are frequently obtained by using natural effects that have rarely or never been used previously in a specific field [14]. The knowledge of various physical, chemical, or geometric effects is necessary for solving a technical problem. Effect interprets the substantial reason for function realization [13], and a function can be performed by an effect or effect chain. The basic ways of establishment of effect chain are called effect patterns, as shown in Fig. 3, in which the directed line segment represents one or several flows [17]. The effect chain can be established by complete matching method, minimal path length method or approximate matching method [18].

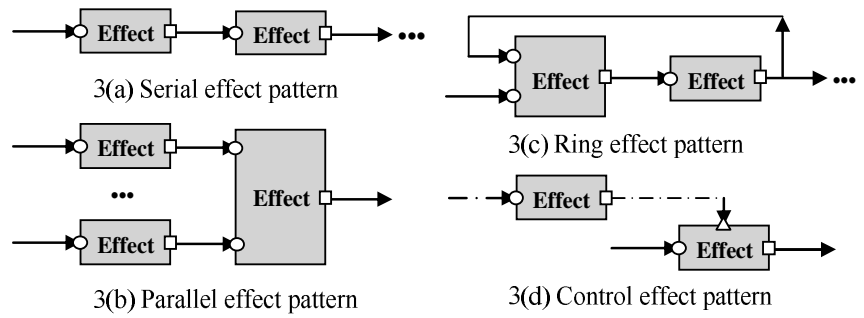

Fig. 3. Effect patterns

In functional design, structure is not concrete structure, but the principle structure (for short, structure), which is a level of abstraction between function and specific elements. Structure is composed of sub-structure, connecting structure, connecting surface, connecting direction and composite mode. According to composite mode, selecting the proper connecting structure, connecting surface and connecting direction, sub-structures are connected to form structure. By the analysis of patents, the structures, which show how the effect is used for the performance of state transitions, can be acquired. An effect can have varied realizing structures, and a structure can be used for varied effects. 
The first step to apply the effects is to develop function structure [6]. Then, one or more sub-functions are selected, for which the solutions need to be found using effects. Some ideas for possible solutions of the sub-functions are searched for using the effect knowledge base. According to effect-structure mapping sub-structures can be confirmed, and product principle structure is constructed by reasonable combination and appropriate adjustment of sub-structures. Fig.4 shows the method.

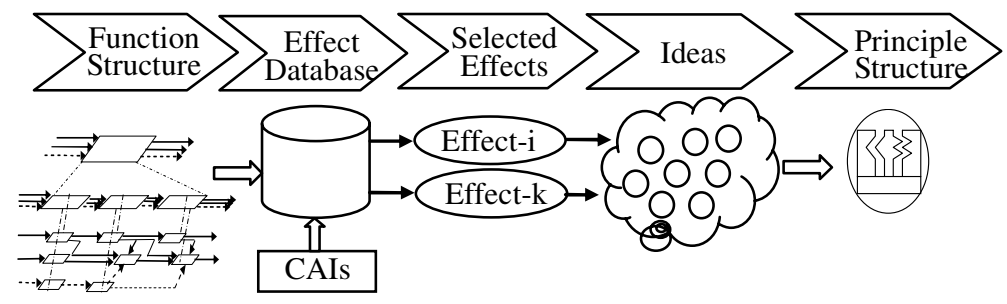

Fig. 4. Principle structure generation using effects

\section{Process Model for Product Integrated Innovation Based on Function}

Function design is to generate principle solution to fulfill the required function. There are several models for functional design process from literatures. References [6-7] are examples of these. In this study Pahl\&Beitz's model [6], as a traditional and humanoriented process model, is selected as a base to extend. Fig. 5 shows a new process model of product integrated innovation design which is supported by computer-aided innovation systems (CAIs) [19].

According to the Fig. 5, the process is divided into 7 steps, which are as following:

Step 1: Establish function structures. For original design [4], the design specification is confirmed according to user requirement, and the overall function is determined by specification. Then the overall function is broken down into a set of sub-functions, which are main function and supporting function of the system that provides minimal working behavior of the system. The performance of system can be improved by function integration and additional functions enhancement. The function structure is established to adapt well to the specifications.

Step 2: Search for working principles for sub-functions. Any complex process of system can be attributed to physical, chemical and logical operations which can be realized by effects. The working principles, including effect and structure, for every sub-function in the existing function structure are found based on function-effect mapping and effect chain in TRIZ, which can be supported by CAIs.

Step 3: Analyze the working principles of sub-functions and find whether there are technical contradictions or not. If there are no contradictions turn to step 6. Otherwise continue the process.

Step 4: Identify contradictions. Analyze all the working principles again and identify all the contradictions clearly. There may be multi-contradictions and they need to identify one by one. 


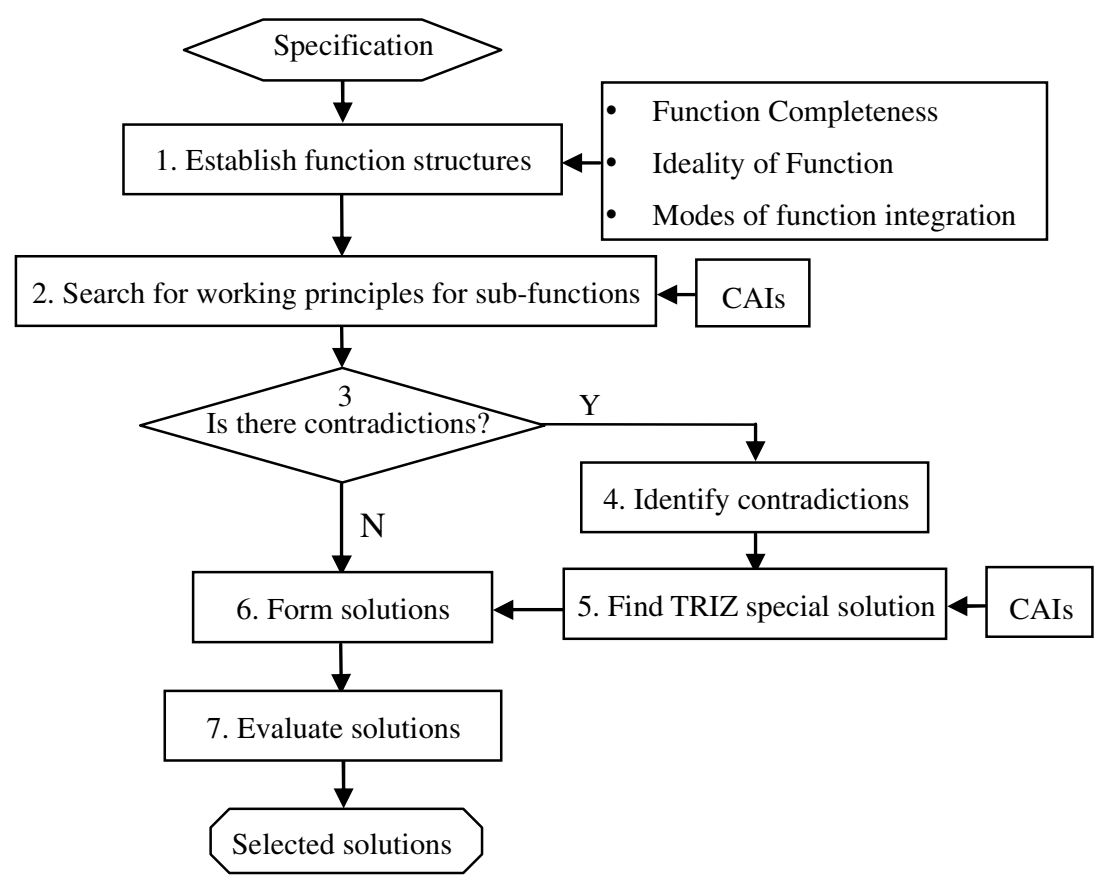

Fig. 5. Process model of product integrated innovation design

Step 5: Find TRIZ special solutions. For each technical contradiction, forty the inventive principles in TRIZ will be applied to solve the problem and the TRIZ special solutions are found under support of CAIs.

Step 6: Form solutions. There are two sub-processes in this step. One is following step 3 and the other is continuing from step 5.

- Combine every working principle of sub-functions into a few system working principles by morphological matrix, named working structures.

- Find new working principles for sub-functions which relate to the contradictions and eliminate the contradictions. Then, combine principles into working structures.

Step 7: Evaluate solutions. Identify one or two working structures as the outputs of functional design process.

\section{Case Study}

Dripping pill is a dosage form prepared by solid dispersing technology, namely, extract of traditional Chinese medicine and solid matrix are molten by heating (range of working temperature: 75-85 ) to form solution, suspension or emulsion, and then droplet is dropped into Coolant which is immiscible with drug matrix. After rapid cooling, the droplet is constricted and condensed to generate dropping pill. In our country the dropping pills machine is still in the primary stage of its development. 
The medicine liquid drips from emitter mainly by its own gravity or the additional air pressure in existing machine. However, the dropping pills in former are various sizes and different weight, and the equipments in latter are complex, high costs and difficult process control. Therefore, a new automatic dropping pills machine should be designed to eliminate the disadvantage of existing machine.

Step 1: Establish or modify function structures.

The overall function of dropping pills machine is to produce geometrical body according to preparation principle of dropping pill, as shown in Fig. 6. According to principle of dropping pill and system integrality, available resources in design are medicament, matrix, edible oil, condensate, electric energy and control parameter, and the output of system to be designed is dropping pill. Therefore the main function of dropping pills machine is to transform droplet, and the supporting functions are supply energy and materials, transport materials, control energy and information. To perform functions better every function can be achieved by several sub-functions, such as the main function is achieved by three sun-functions of move liquid, form droplet and solidify droplet. Thus function structure of dropping pill machine is constructed by function integration, as shown in Fig. 7.

Step 2: Search for working principles for sub-functions.

InventionTool3.0 [20], which is CAIs, is applied in this step. According to the functioneffect mapping the effects that perform functions in Fig. 7 can be determined by effect patterns and reasoning methods in InventionTool3.0, such as the function 'produce heat energy' can be realized by Joule effect, electric arc effect, or Peltier effect, etc. The effect chain for function structure consists of electromotion effect, Joule effect, thermoelectricity effect, heat transfer effect, heat exchange effect, volume displacement effect, surface tension effect, solidification effect and gravity effect.

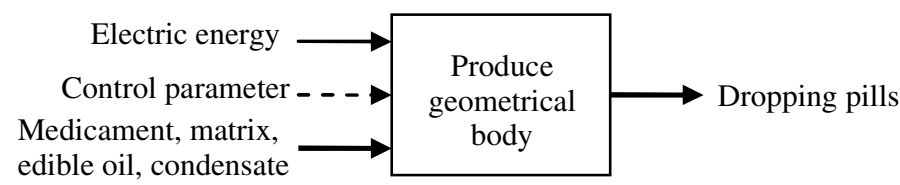

Fig. 6. The overall function of dropping pill machine

Sub-structures which match with effects in effect chain can also be confirmed by CAIs. Taking the function of 'move liquid' as example, the corresponding structures are impeller pump, piston pump, gear pump, magnetic current pump, ultrasound pump, helium pump, electro osmotic pump, peristaltic pump, etc. The peristaltic pump is taken as driving device for dropping system, as shown in Fig. 8. In a similar way, sub-structures corresponding to other sub-functions are confirmed.

According to function structure and combination rule of structure, peristaltic pump, electric heater, etc. are combined into working structure of dropping pills machine [21], as shown in Fig. 9. 


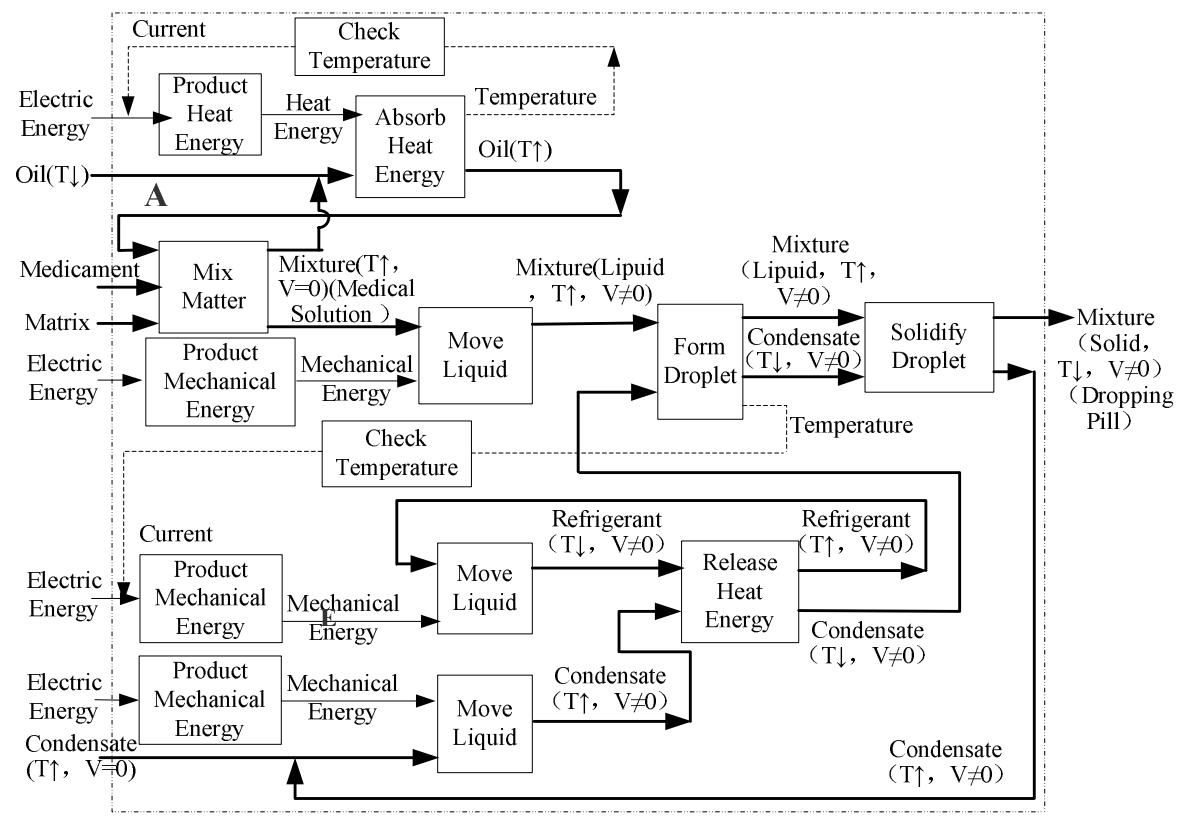

Fig. 7. Function structure of dropping pill machine

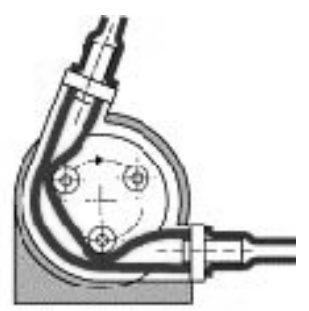

Fig. 8. Schematic diagram of peristaltic pump

Step 3: Find contradictions.

By synthetic valuation of function structure and principle structure, problems of emitter are included as follows: irregularly shaped opening results in bad forming of dropping pill; the amount and specification of flexible pipe are limited; the temperature of flexible pipe is too high to replace manually.

Step 4: Identify contradictions

The problems are defined respectively as technical contradictions: speed (No.9) and manufacturing precision (No.29); manufacturing precision (No.29) and productivity (No.39).

Step 5: Find TRIZ special solutions.

The module 'contradiction solving' in InventionTool3.0 is used to find TRIZ special solutions. Select the improved parameter 'speed' and worse parameter 'manufacturing precision', then, the interfaces show the TRIZ special solutions of this contradiction, 


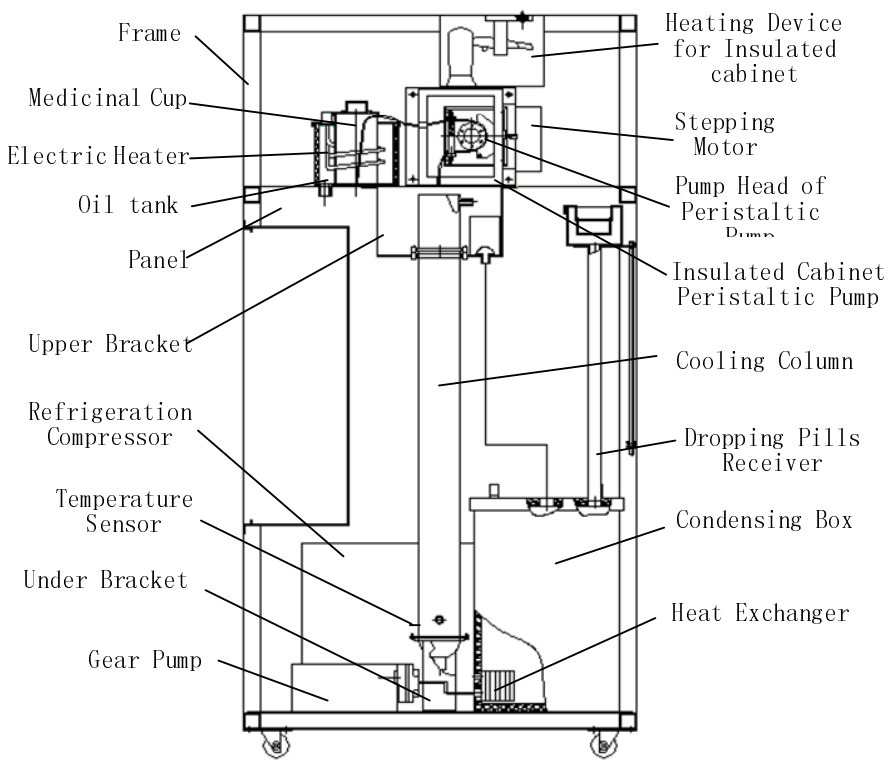

Fig. 9. Schematic construction diagram of dropping pills machine

which are No.25(Self service), No.10(Prior action), No.28(Replacement of mechanical system), No.32(Color change). The four principles and the relevant cases in the case base are the TRIZ special solutions.

By the same process, special solutions of the second contradiction can be found, which are No.18(Vibrate), No.10(Prior action), No.32(Color change), No.39(Inert Atmosphere).

Step 6: Form solutions.

For the solutions of contradictions the designers may generate several ideas by browsing the cases in invetionTool3.0. To resolve the contradictions, a new emitter is developed, as shown in Fig. 10 [22], which has many advantages such as simple in structure, reliable in working, strong in adaptability.

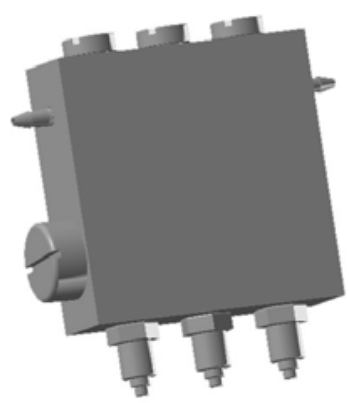

Fig. 10. Emitter assembly diagram 
Step 7: Evaluate solutions.

By synthetic valuation of solution the working structure can fulfill the required function well.

\section{Conclusion}

Product innovation by function integration is an important method for high-level innovation. This paper proposes the process model for product integrated innovation, which provides a systematic approach to product integrated innovation from functional design angle. CAIs show all principles and cases to support the selection and improvement of working structure. The design example for the Chinese dropping pill medicine demonstrates the proposed method is feasible.

\section{Acknowledgments}

This research is supported in part by the Natural Science Foundation of Hebei under Grant Numbers E2008000101, the Key Project of the Ministry of Science and Technology of the People's Republic of China under Grant Numbers 2008IM030100, and the science and technology key project of Hebei Province under Grant Numbers 09212102D. Any opinions or findings of this work are the responsibility of the authors, and do not necessarily reflect the views of the sponsors or collaborators.

\section{References}

1. Jassawalla, A., Sashittal, H.: An Examination of Collaboration in High-technology New Product Development Processes. The Journal of Product Innovation Management 15(3), 124-136 (1998)

2. Marco, I.: Technology Integration: Making Critical Choices in a Dynamic World. Harvard Business School Press (1998)

3. Rothwell, R.: Successful Industrial Innovation: Critical Factors for the 1990s. R\&D Management 22, 221-239 (1992)

4. Boaz, B., Prakash, J.S.: An integrated innovation process model based on practices of Australian biotechnology firms. Technovation 26(5-6), 561-572 (2006)

5. Sarkar, S.: Mapping the Integrated Innovation Space: A Look at the Mirror. PhysicaVerlag HD, Springer (2007)

6. Pahl, G., Beitz, W.: Engineering Design - A Systematic approach, 2nd edn., 3rd Printing. Springer, London (2000)

7. Suh, N.P.: Axiomatic Design: Advances and Applications. Oxford University Press, New York (2001)

8. Jin, Y., Li, W., Lu, S.-Y.: A Hierarchical Co-Evolutionary Approach to Conceptual Design. Proceedings of CIRP Annals-Manufacturing Technology 54(1), 155-158 (2005)

9. Gui, J.K., Mantyla, M.: Functional understanding of assembly modeling. Computer-Aided Design 26(6), 435-451 (1994)

10. Kumara, S.R.T., Kamarthi, S.V.: Function-to-Structure Transformation in Conceptual Design: an Associative Memory-Based Paradigm. Journal of Intelligent Manufacturing 2, 281-292 (1991) 
11. Gero, J.S., Kannengiesser, U.: The situated function-behaviour-structure framework. Artificial Intelligence in Design, 89-104 (2002)

12. Umeda, Y., Ishii, M., Yoshioka, M., et al.: Supporting conceptual design based on the function-behaviour-state modeler. Artificial Intelligence for Engineering Design, Analysis and Manufacturing 10(4), 275-288 (1996)

13. Feng, P.E., Zhang, S., Pan, S.X., et al.: Cyclic solving process and realization for conceptual design of complex function product. Chinese Journal of Mechanical Engineering 41(3), 135-141 (2005) (in Chinese)

14. Altshuller, G.: The Innovation Algorithm, TRIZ, Systematic Innovation and Technical Creativity, Technical Innovation Center, INC, Worcester (1999)

15. Michael, S.S.: Technology Maturity Using S-curve Descriptors. TRIZ Journal (1999), http: / /www.triz-journal.com

16. Valeri, S.: Annotated list of TRIZ techniques (1998), http: / / www. insytec.com/annonsTRIZ.htm

17. Cao, G.Z., Tan, R.H.: Theoretical study and Implementation of Functional Design for Sustainable Innovation. Digital Manufacturing Science 6(3), 24-54 (2008) (in Chinese)

18. Cao, G.Z., Tan, R.H.: FBES Model for Product Conceptual Design. International Journal Product Development 4(1/2), 22-36 (2007)

19. Kohn, S., Husng, S., Kolyla, A.: Development of an Empirical based categorsation scheme for CAI software. In: 1st IFIP TC-5 Working Conference on CAI, ULM, Germany, pp. 143-157 (2005)

20. Tan, R.H., Ma, J.H., Cao, G.Z.: Computer-aided innovation software system: InventionTool 3.0, Software Registration Number: 2006SR13729 (2006)

21. Tan, R.H., Ma, L.H.: An automatic dropping pill machine, Patent Application Number: 200510122183.5 (2005)

22. Sun, J.G., Tan, R.H.: Emitter mechanical module of dropping pill machine, Patent Application Number: 200710056906.5 (2007) 